\title{
MicroRNA-582 promotes tumorigenesis by targeting phosphatase and tensin homologue in colorectal cancer
}

\author{
BIN SONG ${ }^{1,2}$, YANBIN LONG ${ }^{2}$, DONG LIU ${ }^{2}$, WEN ZHANG ${ }^{3}$ and CHANG LIU ${ }^{1}$ \\ ${ }^{1}$ Department of Hepatobiliary Surgery, The First Affiliated Hospital, Xi'an Jiaotong University, Xi'an, \\ Shaanxi 710061; ${ }^{2}$ The Second Department of General Surgery, Shaanxi Provincial People's Hospital; \\ ${ }^{3}$ Department of Otorhinolaryngology, Shaanxi Provincial People's Hospital, Xi'an, Shaanxi 710068, P.R. China
}

Received April 7, 2016; Accepted June 21, 2017

DOI: 10.3892/ijmm.2017.3059

\begin{abstract}
A number of studies have implicated that a class of non-coding RNAs named microRNAs (miRNAs or miRs) is associated with tumorigenesis and have identified miRNAs as promising targets for pharmaceutical intervention. Recently, the deregulated expression of miR-582 in tumor cells has been reported. However, the exact function of miR-582 in colorectal cancer (CRC) remains largely unknown. In thi study, we demonstrate that miR-582 is extensively upregulated in CRC tissues and cell lines. The overexpression of miR-582 significantly enhanced the proliferation and migration ability of the CRC cells. However, the use of a specific miR-582 inhibitor counteracted these effects. miR-582 may also play an oncogenic role by promoting tumor growth in vivo. Phosphatase and tensin homologue deleted on chromosome 10 (PTEN) was identified as a putative target of miR-582; transfection of the cells with a lentivirus with miR-582 mimics substantially decreased both the mRNA and protein levels of PTEN. The restoration of PTEN expression in the CRC cells reversed the adverse effects of miR-582. Our findings therefore indicate that miR-582 promotes CRC progression by decreasing PTEN expression. These findings may also imply that miR-582 may be a target for therapeutic intervention in patients with CRC.
\end{abstract}

\section{Introduction}

Colorectal cancer (CRC) is considered one of the most malignant tumors affecting humans (1). The occurrence of CRC poses a serious threat to life and thousands of individuals succumb to the disease (2). Owing to substantial deficiencies in healthcare and physiological conditions, only a small fraction of patients are eligible for treatment. The onset of CRC has continually

Correspondence to: Dr Chang Liu, Department of Hepatobiliary Surgery, The First Affiliated Hospital, Xi'an Jiaotong University, 277 West Yanta Road, Xi'an, Shaanxi 710061, P.R. China

E-mail: xjtu_liuchang@163.com

Key words: microRNA-582, colorectal cancer, proliferation, migration, phosphatase and tensin homologue increased over the past decades; therefore, more and more attention has been paid to determining effective methods for the earlier diagnosis of CRC (3). However, the overall survival rate for patients with CRC remains low, largely due to the diagnosis being made at a relatively late stage. Therefore, an in-depth understanding of the underlying molecular mechanisms responsible for the disease is of utmost importance for the early diagnosis and potential therapeutic intervention for advanced CRC.

MicroRNAs (miRNAs or miRs) are well-characterized short-length non-coding RNAs capable of suppressing gene expression though base-pairing to the 3 --untranslated regions (3'-UTR) of their targets $(4,5)$. Increasing evidence has suggested that miRNAs play important roles in the occurrence and development of CRC in recent years (6,7). Recently, miR-146 has been shown to direct the symmetric division of spheroid-derived CRC stem cells via feedback loops in Wnt signaling pathways (8). miR-130b is usually downregulated in tumor tissues and inhibits CRC progression by targeting integrin $\beta 1$ (9). Zhang et al recently identified miR-520g as a potential miRNA contributing to multi-drug resistance (10). Further investigation demonstrated that the effect of miR-520g was largely mediated via the regulation of p21 expression (10). Kurihara et al recently provided direct evidence that the expression of a polycomb group protein named enhancer of zeste homolog 2 (EZH2) is highly associated with the expression of miR-31, a well-known oncogenic factor (11).

miR-582 has also been implicated in the progression of specific cancers. For example, the decreased expression of miR-582 has been implicated in the development of bladder cancer and the ectopic expression of miR-582-5p can inhibit the migration and invasion of bladder cancer cell lines (12). Another study also demonstrated that miR-582-5p was significantly upregulated in patients with tuberculosis and functioned as an anti-apoptotic factor (13). However, little information is available on the exact role of miR-582 in CRC.

Phosphatase and tensin homologue deleted on chromosome $10(P T E N)$ is a tumor-suppressor gene located on human chromosome 10q23.3, which is both a lipid phosphatase and a protein phosphatase (14). The inactivation of PTEN results in the constitutive activation of the PI3K/AKT pathway with increased proliferation and survival (15). Of note, as previously demonstrated, the loss of PTEN may promote the progression 
of CRC and PTEN can be used as a predictor of the outcome following treatment with bevacizumab (16). Therefore, PTEN may be critically involved in the pathogenesis of CRC.

In the present study, we found that the expression of miR-582 was frequently upregulated in CRC cell lines, as well as human specimens. In addition, the overexpression of miR-582 significantly promoted the colony-forming and the migration ability of HCT-116 cells. The adverse effects of the overexpression of miR-582 were partially reversed by synthetic miR-582 inhibitor. The systematic identification of miR-582 targets suggested that PTEN may be a direct target. The expression of PTEN transcript and protein was substantially inhibited by the overexpression of miR-582. miR-582 also promoted tumor xenograft growth and downregulated PTEN expression. Therefore, our results suggest that miR-582 may serve as a putative target for cancer intervention by dynamically regulating PTEN.

\section{Materials and methods}

Human samples and cell lines. The CRC cell lines used in the present study (HCT-116, SW-480, SW-620, SW-948, DLD-1 and HT-29) were all commercially obtained from the American Type Culture Collection (ATCC; Rockville, MD, USA). The control epithelial colon cell line, FHC, was also purchased from ATCC. These cells were cultured in RPMI-1640 medium (Gibson, Gaithersburg, MD, USA). The medium was supplemented with $3 \%$ fetal bovine serum (FBS) and penicillin $(200 \mathrm{U} / \mathrm{ml})$ (both from Gibson) in a humidified atmosphere $5 \% \mathrm{CO}_{2}$ at $37^{\circ} \mathrm{C}$. The CRC specimens and adjacent normal tissues $(n=83)$ were all surgical archives from patients at the First Affiliated Hospital, Xi'an Jiaotong University (Shaanxi, China) obtained between October 2013 and June 2015. All specimens were kept in liquid nitrogen at $-80^{\circ} \mathrm{C}$ following resection prior to use in the experiments. All patients provided formal and signed consent forms. The protocols of the experimental procedures for the use of human samples were formally approved by the Research Ethics Committee of the First Affiliated Hospital, Xi'an Jiaotong University (no. 2013H015).

Target prediction. We used algorithms for target gene prediction TargetScan (http://genes.mit.edu/targetscan), and miRDB (www.mirdb.org), as previously described $(17,18)$. Briefly, putative targets were ranked by $\mathrm{z}$ scores. The top ranked overlapping targets were selected for experimental verification.

$R N A$ extraction and reverse transcription-quantitative PCR (RT-qPCR). The gene expression of miR-582 and PTEN in the CRC cells and tissues was measured by RT-qPCR. In brief, RNA was extracted from both the CRC cells and CRC human specimens using TRIzol reagent (Invitrogen Life Technologies, Carlsbad, CA, USA) and reverse transcribed into cDNA using the miScript II RT kit (Qiagen GmbH, Hilden, Germany) according to the instructions provided by the manufacturer. The corresponding cDNA was then used as a template for qPCR performed using the SYBR Premix Ex Taq ${ }^{\mathrm{TM}}$ kit (Takara Bio, Inc., Otsu, Japan) according to the manufacturer's instructions. A TaqMan miRNA qRT-PCR kit (Applied Biosystems, Foster City, CA, USA) was applied and GAPDH was used as a control. For PTEN detection, we used the SYBR-Green PCR Master Mix kit (Applied Biosystems). All kinetic reactions for RT-qPCR were carried out using the ABI Prism ${ }^{\circledR} 7000$ Sequence Detection System (Applied Biosystems). Relative expression was determined. The primer sequences were as follows: PTEN forward, 5'-AGCAATGTT AAGCCGAG-3' and reverse, 5'-TACGCCCGCACTA TTGAGTAGCA-3'; miR-582 forward, 5'-ACACTCGAGCT GGGGCTTGTTCGCTAGCATT-3' and reverse, 5'-TGATG CTTCTGAGTCG-3'; and GAPDH forward, 5'-CTCGATCTT CATAGCGCGTCG-3' and reverse, 5'-ATGTCGTTCTCA GCCTTGAC-3'.

Cell line transfection. The lentiviral system to ectopically overexpress miR-582 in the HCT-116 cell line was used in the present study. The lentiviruses with miR-582 mimics (Lenti-miR-582) and negative controls (Lenti-NC) were synthesized and purchased both from Sigma-Aldrich (Shanghai, China). The miR-582 precursor, miR-582 inhibitor and the scramble control were all obtained from Sigma-Aldrich. The pcDNA3.1-PTEN plasmids were used for transfection. A scramble pcDNA3.1 plasmid was used as a control. The Lipofectamine ${ }^{\mathrm{TM}} 2000$ system (Invitrogen, Shanghai, China) was used for viral transfection. At $24 \mathrm{~h}$ post-transfection, the culture medium was replaced with fresh medium. The transfection efficiency of all plasmids was experimentally verified by RT-qPCR.

Dual luciferase reporter assay. The recombinant psi-CHECKrcmiR-582-WT and psiCHECK-PTEN-3'-UTR-WT vectors were constructed by linking the seed sequences with the 3'-UTR of PTEN or reverse complement of miR-582 ( $\mathrm{rcmiR}-582$ ). The combined sequences were inserted into the psi-CHECK vector. Mutant constructs were similarly obtained. The mutation spots were indicated. The constructs were verified by DNA sequencing. 293T cells (Institute of Biochemistry and Cell Biology, Shanghai, China) were loaded into a 96-well plate $24 \mathrm{~h}$ prior to transfection and then co-transfected with $1 \mu \mathrm{g}$ recombinant vectors alone or vectors with additional $20 \mathrm{nM}$ precursors or inhibitors. Transfection was carried out using the Lipofectamine 2000 system (Invitrogen). The luciferase activities were measured using the Dual-Luciferase reporter system (Promega, Madison, WI, USA) as relative luciferase units following the manufacturer's instructions.

Wound healing and migration assay. The wound healing assay was used to quantify the migration ability of the cancer cells. The experiments were performed as previously described (19). Briefly for wound healing assay, the HCT-116 cells were seeded on $25-\mathrm{mm}$ dishes and cultured in RPMI-1640 medium (Gibson). A scratch with a fixed width was formed in the cell monolayer using a $200 \mu \mathrm{l}$ pipette tip. Subsequently, all cells were washed twice with fresh phosphate-buffered saline (PBS) and the suspended ones were then removed. Wound healing was monitored and images were acquired at 0 and $48 \mathrm{~h}$ after the scratch was made using a bright-field microscope (Olympus, Tokyo, Japan).

Proliferation assay. The HCT-116 cells were seeded in 96-well plates $\left(10^{4}\right.$ cells/well) for 5 days. The Cell Counting Kit-8 (CCK-8; Dojindo Laboratories, Kumamoto, Japan) was used. At an interval of $24 \mathrm{~h}$, the 3-(4,5-dimethylthi- 
A

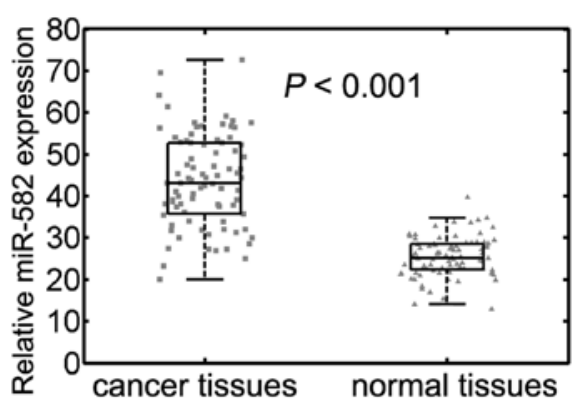

B

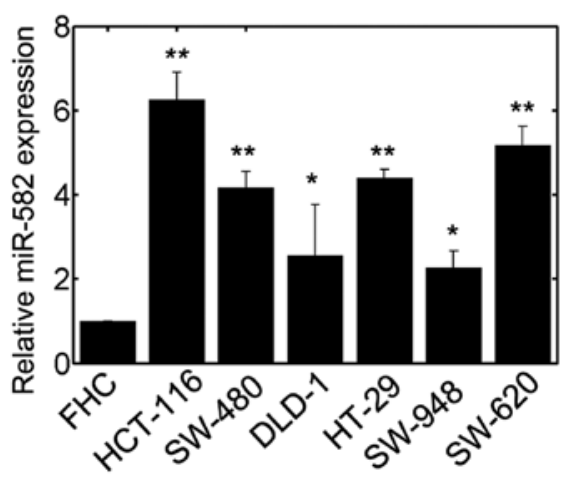

Figure 1. miR-582 is upregulated in colorectal cancer (CRC). (A) The expression of miR-582 in 83 specimens compared with normal adjacent tissues (P<0.001). (B) The expression of miR-582 in normal colon epithelial cell (FHC) and CRC cell lines (HCT-116, SW-480, SW-620, SW-948, DLD-1 and HT-29). The expression of miR-582 was normalized to endogenous GAPDH. Error bars denote the means $\pm \mathrm{SD}$. ${ }^{*} \mathrm{P}<0.05,{ }^{* *} \mathrm{P}<0.01$ vs. normal cell line.

azol-2-yl)-2,5-diphenyltetrazolium bromide (MTT) solution was added to the culture medium at a final concentration of $5 \mathrm{mg} / \mathrm{ml}$. After $6 \mathrm{~h}$, the medium was removed and crystalline formazan was dissolved in $100 \mu \mathrm{l}$ SDS (10\%; Gibson) solution for 1 day. The optical density (OD, $490 \mathrm{~nm}$ ) was evaluated using a SpectraMax M5 microplate reader (Molecular Devices, Sunnyvale, CA, USA).

Colony formation assay. The cells were trypsinized to obtain a single cell suspension. Subsequenlty, 400 HCT-116 cells were seeded in a 12-well plate and the medium was refreshed with fresh medium every 2 days. After 6 days, the medium was discarded and the cells were stained with crystal violet (Sigma-Aldrich, Shanghai, China) (0.2\% in $20 \%$ methanol). Images were acquired using a digital camera.

Xenograft implantation. In total, $10^{7} \mathrm{HCT}-116$ cells transfected with the empty vector control, lentiviral miR-582 or Lenti-NC were injected subcutaneously into BALB/c nude mice into the right flank. In total, 27 mice (aged 4-5 weeks; average weight, $19.6 \mathrm{~g}$; males, 0; females, 27) were used. The mice were obtained from the Model Animal Research Center (Nanjing, China). The mice were housed in an environment with a temperature of $20^{\circ} \mathrm{C}, 55-60 \%$ humidity and a light-dark cycle of $12 \mathrm{~h}$. The access to food and water was provided ad libitum. The protocols for the current study (no. 15-006) were approved by the Animal Research Committee of the First Affiliated Hospital of Xi'an Jiaotong University (Xi'an, China). The mice were divided into 3 groups ( $n=8$ per group) according to the transfected cells injected. The tumor volume was measured at an interval of 3 days for a total of 30 days. By the end of the implantation, all mice were sacrificed.

Immunochemistry. All specimens were fixed with $15 \%$ formalin and loaded in paraffin. The sections (2- $\mu$ m-thick) were then examined. Deparaffinized sections were then subjected to antigen retrieval by autoclaving in $20 \mathrm{mmol} / \mathrm{l}$ citrate buffer $(\mathrm{pH} 6.0)$ at $120^{\circ} \mathrm{C}$ for $15 \mathrm{~min}$. The sections were immunostained using the Histofine stain kit (Nichirei, Tokyo, Japan). The antibody against PTEN (Cat. no. P3487) used in this study was diluted at a ratio of 1:100 (Sigma-Aldrich). The stained sections were thyen visualized with diaminobenzidine and counterstained with hematoxylin.

Western blot analysis. The HCT-116 cells were harvested with lysis buffer (10\% glycerol and 3\% NP-40) obtained from Sigma-Aldrich. The protein extracts (equally $100 \mu \mathrm{g}$ for each) were dissolved in 10\% SDS-PAGE and transferred onto nitrocellulose membranes (Bio-Rad Laboratories, Inc., Hercules, CA, USA). The membranes were then coated with monoclonal anti-PTEN (Cat. no. P3487) and anti-GAPDH (Cat. no. G8795) antibodies (both from Sigma-Aldrich) overnight. The HRP-conjugated secondary antibodies $(1: 1,000)$ were then added followed by incubation at $20^{\circ} \mathrm{C}$ for $1.5 \mathrm{~h}$. The blots were then visualized using the chemiluminescence film system (Amersham Pharmacia Biotech, Shanghai, China).

Statistical analysis. The results were all analyzed using SPSS version 15.0 software (SPSS, Inc., Chicago, IL, USA). All experiments were carried out at least 3 times. P-values $<0.05$ were considered to indicate statistically significant differences. A paired U test was used for pair-wise comparisons.

\section{Results}

miR-582 is frequently upregulated in human CRC specimens and cell lines. We aimed to determine whether miR-582 plays a role in CRC. We compared the expression of miR-582 in 83 cases of CRC tissues and adjacent normal tissues using RT-qPCR. The results revealed that the miR-582 level was substantially increased in the cancer tissues compared with the adjacent normal tissues (Fig. 1A). We also quantified the expression of miR-582 in 6 well-characterized CRC cell lines, as well as in a normal epithelial colon cell line. The results revealed that miR-582 was also upregulated in the CRC cell lines (Fig. 1B). These results suggested that miR-582 was ubiquitously elevated in CRC in comparison with the normal controls. The upregulation of miR-582 may also suggest that miR-582 contributes to the progression of CRC. Since the HCT-116 cells displayed the relatively highest expression of miR-582, we selected the HCT-116 cell line for further in vitro analysis. 
A

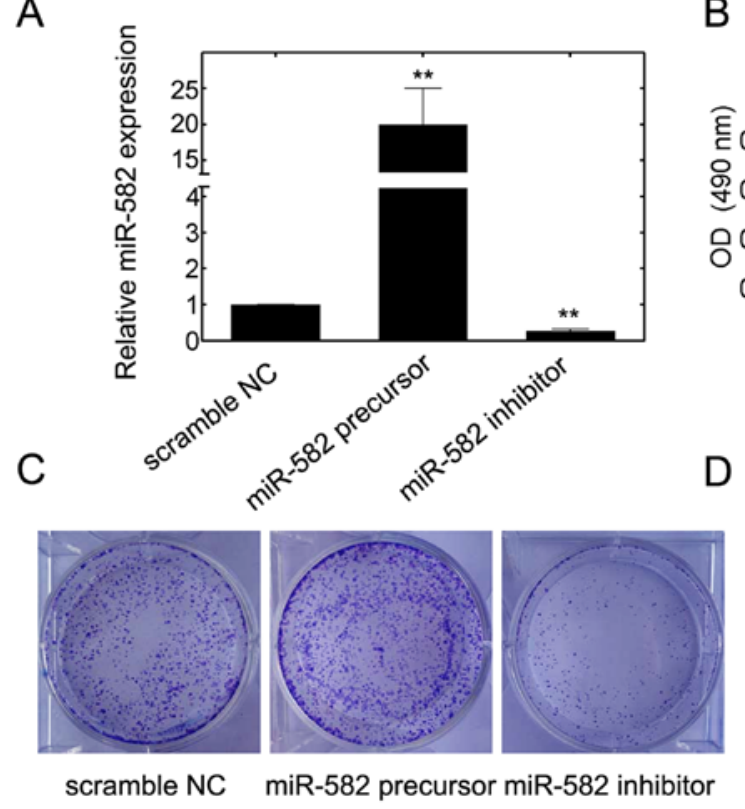

B

D
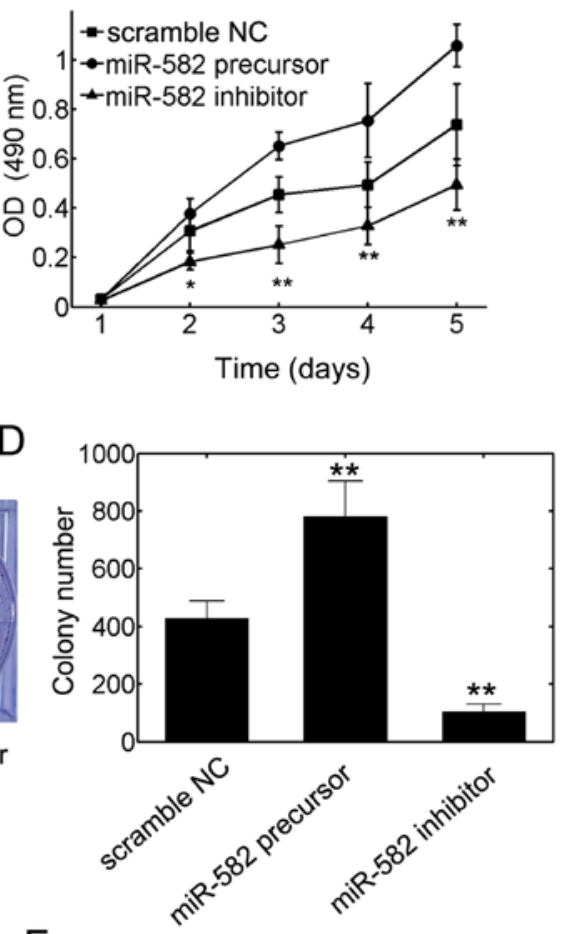

F

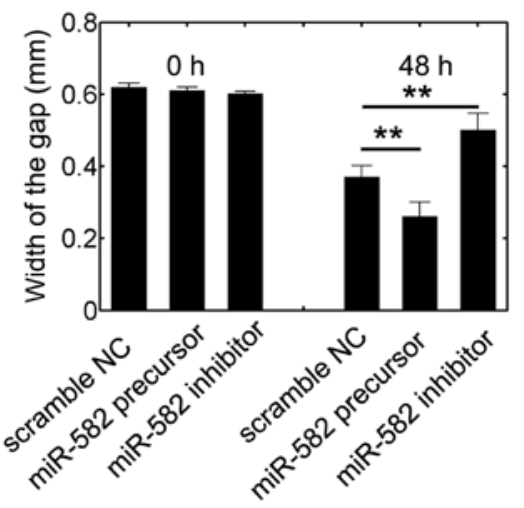

Figure 2. Effect of miR-582 in vitro. (A) The expression of miR-582 in HCT-116 cells transfected with scramble NC, miR-582 precursor or miR-582 inhibitor. The results were quantified by RT-qPCR. (B) MTT assay for the proliferation of HCT-116 cells transfected with scramble NC, miR-582 precursor or miR-582 inhibitor. (C) Colony formation assay for HCT-116 cells. (D) Quantification of results in (C). (E) The migratory capacity of HCT-116 cells was determined by wound healing assay. Wound healing was evaluated at $48 \mathrm{~h}$ after the scratch was made. (F) Quantification of the results in (E). Error bars denote the means \pm SD. ${ }^{*} \mathrm{P}<0.05,{ }^{* *} \mathrm{P}<0.01$ vs. the scramble control (NC).

miR-582 enhances the malignant potential of CRC cells. To verify whether miR-582 has a direct affect on CRC cell proliferation, we transfected the HCT-116 cells with miR-582 precursor or inhibitor. The efficiency of transfection was verified (Fig. 2A). The results of MTT assay revealed that the overexpression of miR-582 markedly increased cell proliferation (Fig. 2B). However, using miR-582 specific inhibitors, we found that the adverse effects of miR-582 transfection were effectively reversed (Fig. 2B). Subsequently, colony formation assays were performed. The result revealed that transfection with miR-582 precursor significantly increased the number of colonies (Fig. 3C and D). However, miR-582 inhibitor markedly decreased colony formation compared with the controls (Fig. 3C and D). Finally, wound healing assay was used to evaluate the migration ability of the CRC cells. The results revealed that the migratory capacity of the tumor cells was substantially enhanced following transfection with
miR-582 precursor, as evidenced by the decreased wound width (Fig. 2E and F). We also confirmed the efficacy of miR-582 inhibitor transfection owing to the significant reduction in migration (Fig. 2E and F). These results collectively suggest that miR-582 enhances the proliferative and migration ability of the CRC cells.

Identification of the potential target of miR-582. miRNAs can post-transcriptionally regulate gene expression and exert their biological effects (20). In this study, to identify the molecular mechanisms of action of miR-582 in CRC, we used multiple algorithms to predict the target of miR-582 (microRNA.org, http://www.microrna.org/microrna/home.do and MIRDB, www.mirdb.org). It was suggested that PTEN may be a direct target. To confirm the targeting effect of miR-582, luciferase reporters were used. Vectors containing the seed sequences were used (3'-UTR) (Fig. 3A). The psiCHECK 


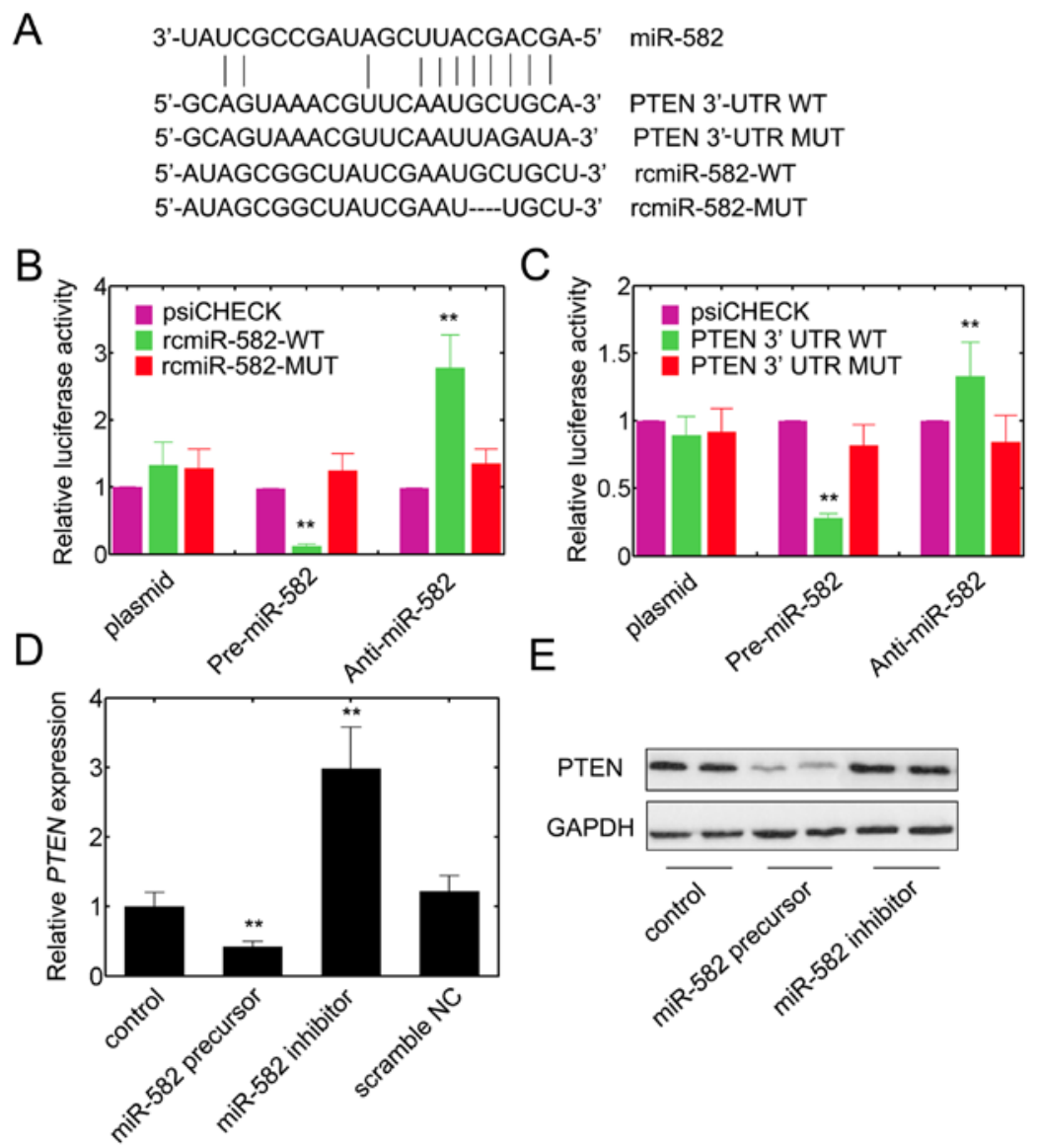

Figure 3. Identification of the target of miR-582. (A) Alignment of miR-582 with rcmiR-582 (rcmiR-582-WT), PTEN (PTEN-3'-UTR WT), mutant rcmiR-582 (rcmiR-582-MUT) and mutant PTEN (PTEN-3'-UTR MUT). (B) Dual luciferase activity using empty plasmids alone or in combination with miR582 precursor and miR-582 inhibitor. The rcmiR-582-WT and rcmiR-582-MUT were used as controls. (C) Dual luciferase activity using empty plasmids alone or in combination with miR-582 precursor and miR-582 inhibitor. The PTEN-3'-UTR WT or PTEN-3'-UTR MUT were used separately. (D) The mRNA level of PTEN was determined by RT-qPCR. (E) The protein expression of PTEN was evaluated by western blot analysis. GAPDH was used as a control. Error bars denote the means $\pm \mathrm{SD}$. ${ }^{* *} \mathrm{P}<0.01$ vs. control.
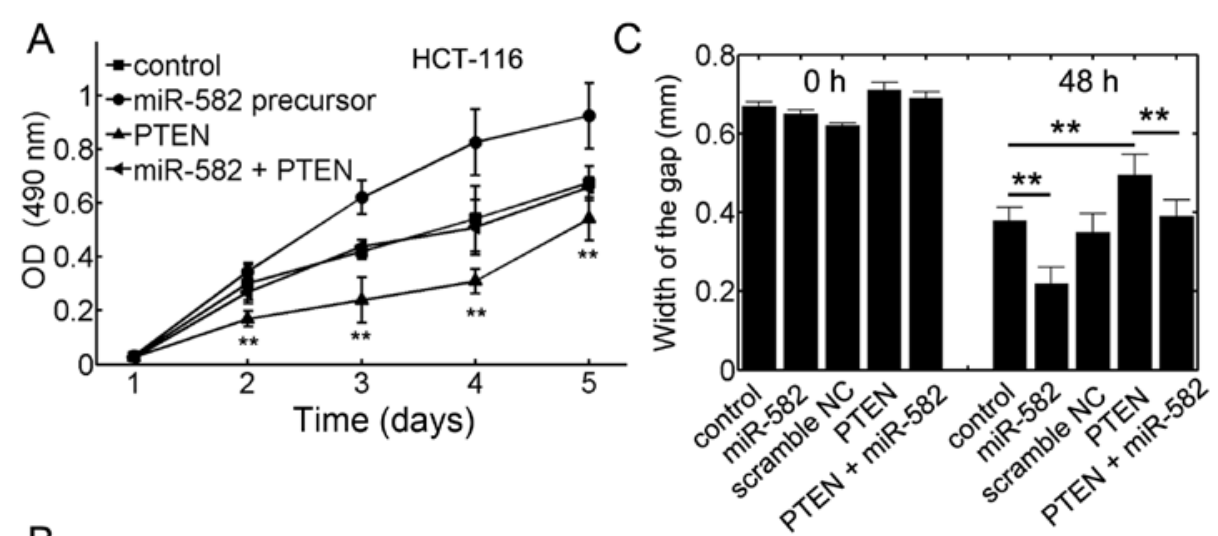

B
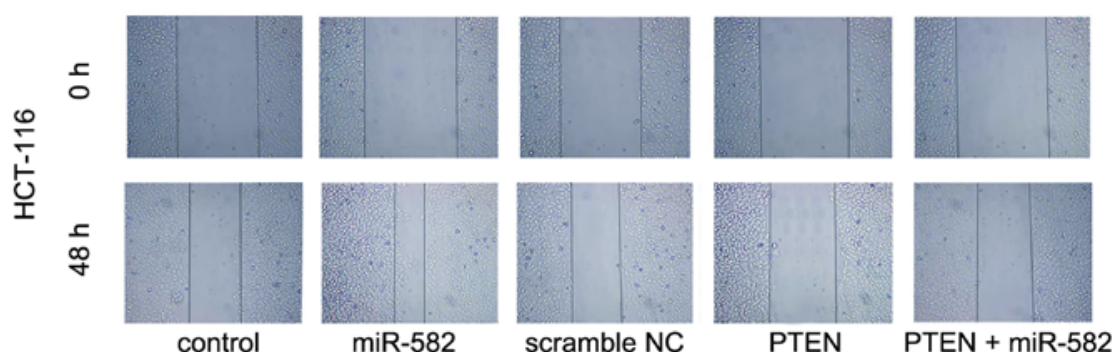

Figure 4. miR-582 exerts its effects by targeting PTEN. (A) MTT assay was performed to evaluate the proliferation of transfected HCT-116 cells as indicated. (B) Wound healing assays for HCT-116 migration. Wound healing was determined at $48 \mathrm{~h}$ after the scratch was made. The width of the gap was determined to evaluate cell migration. (C) The results in (B) were quantified. Statistical significance is shown, ${ }^{* *} \mathrm{P}<0.01$ vs. control. 
A

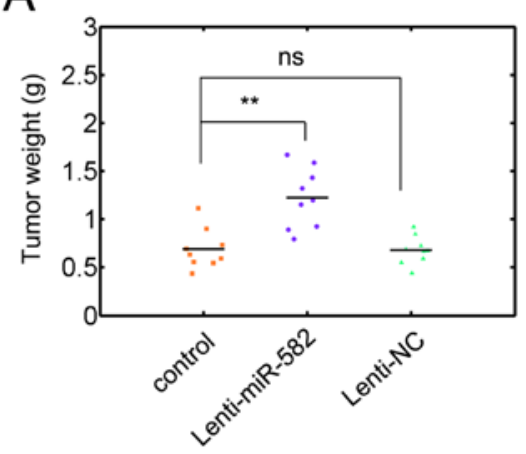

$B$

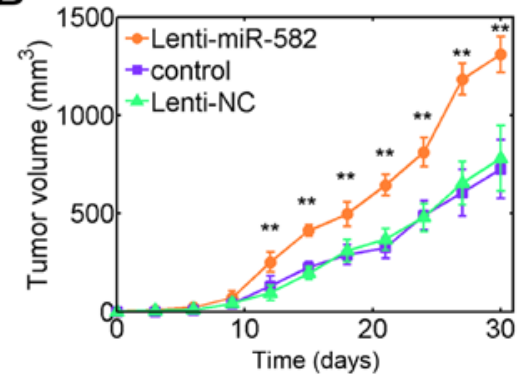

C

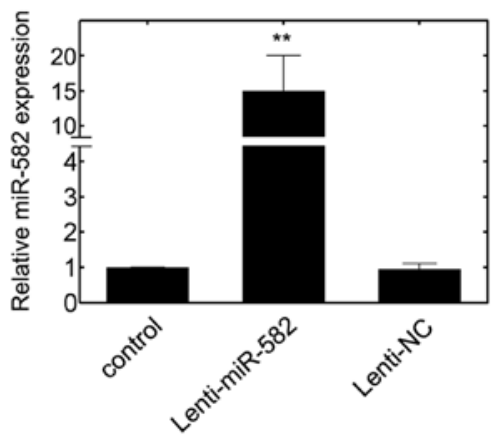

D

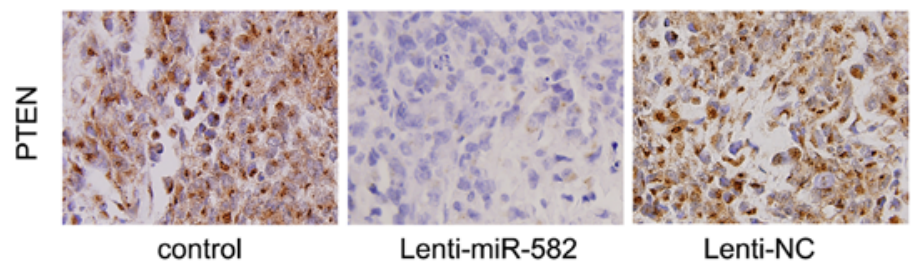

Figure 5. miR-582 promotes the growth of colorectal cancer (CRC) xenografts. (A) Xenograft tumors were induced by injecting HCT-116 cells into nude mice. Each group contained 9 mice. The mean values are shown as horizontal lines. (B) The tumor volumes were determined every 3 days for a total of 30 days (C) Relative expression of miR-582 in xenografts was evaluated by RT-qPCR. (D) Immunohistochemistry for PTEN expression in various groups. Error bars denote the means $\pm \mathrm{SD} .{ }^{* *} \mathrm{P}<0.01$ vs. control; ns, not significant.

empty vector (negative control) and reverse complementary miR-582 (rcmiR-582, positive control) were utilized (Fig. 3A). The efficiencies for positive and negative controls were verified (Fig. 3B). The results revealed that miR-582 precursor significantly inhibit the luciferase activities of the constructs containing PTEN 3'-UTR WT (Fig. 3C). However, the effect of miR-582 was severely reduced when the 3'-UTR of PTEN was mutated (Fig. 3C). Moreover, miR-582 can directly target PTEN in CRC cells, as miR-582 was found to decrease the transcripts of PTEN (Fig. 3D). The protein level of PTEN was also downregulated following transfection with miR-582 precursor (Fig. 3E). Transfection with miR-582 inhibitor reversed the effects of miR-582 on PTEN expression at both the mRNA and protein level (Fig. 3D and E). These results thus suggest that miR-582 directly targets PTEN for tumorigenesis.

miR-582 targets PTEN to promote malignant phenotypes in $C R C$. To further evaluate the effects of miR-582 in CRC, we transfected the HCT-116 cells with pcDNA3.1-PTEN alone or together with miR-582 precursor. The results revealed that transfection with pcDNA3.1-PTEN substantially decreased the proliferation of the HCT-116 cells (Fig. 4A). However, the effect of pcDNA3.1-PTEN transfection was counteracted by transfection with miR-582 precursor (Fig. 4A). Wound healing assays also demonstrated that the migratory ability of the HCT-116 cells was decreased by transfection with pcDNA3.1PTEN (Fig. 4B and C). Consistently, transfection with miR-582 precursor promoted cell migration (Fig. 4B and C). However, the adverse effects of transfection with miR-582 precursor were somehow neutralized by co-transfection with pcDNA3.1-PTEN, as co-transfection with miR-582 precursor and pcDNA3.1$P T E N$ increased cell migration compared to that observed with transfection with pcDNA3.1-PTEN alone, but not to the extent observed with miR-582 precursor transfection (Fig. 4B and C). These data suggest that miR-582 promotes the progression of CRC by directly targeting PTEN.

miR-582 promotes tumor growth in vivo. To further verify the effects of miR-582 in vivo, HCT-116 cells were first transfected with empty control, Lenti-NC or Lenti-miR-582. Subsequently, the HCT-116 cells were subcutaneously injected into nude mice into the right flank. The xenograft model comprised of 3 groups each containing 9 mice. The tumor volume was monitored every 3 days. After 30 days, all mice were sacrificed.

We found that the injection of cells transfected with Lenti-miR-582 led to a significantly increased tumor weight $(\mathrm{P}<0.01$; Fig. $5 \mathrm{~A})$. Moreover, the growth rate of the Lenti-miR-582-transfected xenografts was also substan- 
tially increased compared with the control or Lenti-NC groups (Fig. 5B). The level of miR-582 was also stable at 30 days post-injection (Fig. 5C). Furthermore, immunohistochemistry also confirmed a decreased PTEN expression in the tumor xenografts formed from the Lenti-miR-582-transfected cells (Fig. 5D). These results demonstrate that miR-582 functions as an oncogenic factor in CRC by targeting PTEN.

\section{Discussion}

miRNAs are a class of non-coding RNAs and are critically involved in various biological processes (21). miRNAs can regulate tumor occurrence and progression by directly targeting numerous genes associated with oncogenesis, tumor suppression, proliferation or apoptosis (22). Deregulated miRNA expression always plays a pivotal role in tumorigenesis in many types of cancer (23-25). Therefore, the understanding of the association between miRNAs and tumorigenic factors may shed light on more effective therapeutic intervention.

A recent study demonstrated that miR-582-5p inhibits bladder cancer progression (12). A more recent study suggested that miR-582 targets Rab27a and promotes tumor cell progression, although their findings were not conclusive (26). In glioblastoma stem cells, miR-582-5p was shown to inhibit apoptosis by targeting caspase-9, caspase-3 and possibly Bim (27). Therefore, the exact function of miR-582, particularly in CRC remains largely elusive. In this study, we report that miR-582 targets PTEN and promote tumorigenesis in CRC. Both in vitro and in vivo experiments demonstrated that miR-582 increased the oncogenic potential of CRC cells. The inhibition of miR-582 expression by transfection with miR-582 inhibitor counteracted the adverse effects of CRC cells, suggesting that targeting miR-582 may provide a potentially effective strategy with which to annihilate malignant tumors, particularly CRC. Notably, miRNAs can both function as oncogenic factors or tumor suppressors in a cell typespecific manner. For example, Jiang et al found that miR-492 promoted tumorigenesis in hepatocarcinoma by targeting PTEN (28). However, the decreased expression of miR-492 was also shown to contribute to oxaliplatin resistance by increasing CD147 expression in colon cancer cell lines, implying that miR-492 may serve as a tumor suppressor (29). These findings suggest that even the same miRNA can play different roles under different genetic backgrounds.

miRNA-based therapeutics have been extensively reported in recent years. For example, the systematic delivery of miR-34a has been proven to be effective in inhibiting lung cancer (30). The administration of formulated miRNAs has also been proven not to significantly affect blood chemistry, guaranteeing the safety of miRNA delivery (30). In addition, recent study demonstrated that the delivery of synthetic miR-143 inhibited the migration of osteosarcoma (31). A number of studies have suggested that the delivery of miRNAs alters the tumorigenic potential of various tumors (32-35). Given the role of miR-582 in the progression of $\mathrm{CRC}$, the systematic delivery of miR-582 inhibitor may provide an alternative method with which to inhibit tumorigenesis.

PTEN is an important tumor suppressor gene and various miRNAs can target PTEN to regulate tumorigenesis $(28,36-38)$. PTEN is decreased in patients with cancer and its deficiency is usually associated with tumorigenesis (39). Accumulating evidence has suggested that PTEN inhibits tumor progression by abolishing the PI3K/AKT pathway through the dephosphorylation of PIP3 (40). Moreover, PTEN has also been implicated in a positive feedback loop mediated through the tumor suppressor p53 (41). In this study, we confirmed that PTEN may be a direct target of miR-582 via multiple strategies. Therefore, miR-582 may also participate in the regulation of other tumor suppressor pathways via intrinsic feedback loops. These implications may provide the basis for designing effective miR-582 inhibitors for the targeting of miR-582 to promote tumor suppression.

In conclusion, our study demonstrates that miR-582 promotes CRC progression and serves as an oncogenic factor. $P T E N$ was predicted to be the direct target of miR-582. By regulating PTEN expression, miR-582 substantially increases the proliferation and migration of CRC cells. Our findings suggest that miR-582 may serve as a potential target for CRC intervention in the future.

\section{References}

1. Böckelman C, Engelmann BE, Kaprio T, Hansen TF and Glimelius B: Risk of recurrence in patients with colon cancer stage II and III: a systematic review and meta-analysis of recent literature. Acta Oncol 54: 5-16, 2015.

2. Akiyoshi T, Kobunai T and Watanabe T: Recent approaches to identifying biomarkers for high-risk stage II colon cancer. Surg Today 42: 1037-1045, 2012.

3. Torre LA, Bray F, Siegel RL, Ferlay J, Lortet-Tieulent J and Jemal A: Global cancer statistics, 2012. CA Cancer J Clin 65: 87-108, 2015.

4. Miska EA: How microRNAs control cell division, differentiation and death. Curr Opin Genet Dev 15: 563-568, 2005.

5. Cai Y, Yu X, Hu S and Yu J: A brief review on the mechanisms of miRNA regulation. Genomics Proteomics Bioinformatics 7: 147-154, 2009.

6. Ogata-Kawata H, Izumiya M, Kurioka D, Honma Y, Yamada Y, Furuta K, Gunji T, Ohta H, Okamoto H, Sonoda H, et al: Circulating exosomal microRNAs as biomarkers of colon cancer. PLoS One 9: e92921, 2014.

7. Shivapurkar N, Weiner LM, Marshall JL, Madhavan S, Deslattes Mays A, Juhl H and Wellstein A: Recurrence of early stage colon cancer predicted by expression pattern of circulating microRNAs. PLoS One 9: e84686, 2014.

8. Hwang WL, Jiang JK, Yang SH, Huang TS, Lan HY, Teng HW, Yang CY, Tsai YP, Lin CH, Wang HW, et al: MicroRNA-146a directs the symmetric division of Snail-dominant colorectal cancer stem cells. Nat Cell Biol 16: 268-280, 2014.

9. Zhao Y, Miao G, Li Y, Isaji T, Gu J, Li J and Qi R: MicroRNA- 130b suppresses migration and invasion of colorectal cancer cells through downregulation of integrin $\beta 1$ [corrected]. PLoS One 9: e87938, 2014.

10. Zhang Y, Geng L, Talmon G and Wang J: MicroRNA-520g confers drug resistance by regulating p21 expression in colorectal cancer. J Biol Chem 290: 6215-6225, 2015.

11. Kurihara H, Maruyama R, Ishiguro K, Kanno S, Yamamoto I, Ishigami K, Mitsuhashi K, Igarashi $\mathrm{H}$, Ito M, Tanuma T, et al: The relationship between EZH2 expression and microRNA-31 in colorectal cancer and the role in evolution of the serrated pathway. Oncotarget 7: 12704-12717, 2016.

12. Uchino K, Takeshita F, Takahashi RU, Kosaka N, Fujiwara K, Naruoka H, Sonoke S, Yano J, Sasaki H, Nozawa S, et al: Therapeutic effects of microRNA-582-5p and $-3 p$ on the inhibition of bladder cancer progression. Mol Ther 21: 610-619, 2013.

13. Liu Y, Jiang J, Wang X, Zhai F and Cheng X: miR-582-5p is upregulated in patients with active tuberculosis and inhibits apoptosis of monocytes by targeting FOXO1. PLoS One 8: e78381, 2013.

14. Saal LH, Gruvberger-Saal SK, Persson C, Lövgren K, Jumppanen M, Staaf J, Jönsson G, Pires MM, Maurer M, Holm K, et al: Recurrent gross mutations of the PTEN tumor suppressor gene in breast cancers with deficient DSB repair. Nat Genet 40: 102-107, 2008. 
15. Di Cristofano A and Pandolfi PP: The multiple roles of PTEN in tumor suppression. Cell 100: 387-390, 2000.

16. Price TJ, Hardingham JE, Lee CK, Townsend AR, Wrin JW, Wilson K, Weickhardt A, Simes RJ, Murone C and Tebbutt NC: Prognostic impact and the relevance of PTEN copy number alterations in patients with advanced colorectal cancer (CRC) receiving bevacizumab. Cancer Med 2: 277-285, 2013.

17. Lewis BP, Shih IH, Jones-Rhoades MW, Bartel DP and Burge CB: Prediction of mammalian microRNA targets. Cell 115: 787-798, 2003.

18. Wong $\mathrm{N}$ and Wang $\mathrm{X}$ : miRDB: an online resource for microRNA target prediction and functional annotations. Nucleic Acids Res 43: D146-152, 2015.

19. Han XJ, Yang ZJ, Jiang LP, Wei YF, Liao MF, Qian Y, Li Y, Huang X, Wang JB, Xin HB, et al: Mitochondrial dynamics regulates hypoxia-induced migration and antineoplastic activity of cisplatin in breast cancer cells. Int J Oncol 46: 691-700, 2015.

20. Cheng CJ, Bahal R, Babar IA, Pincus Z, Barrera F, Liu C, Svoronos A, Braddock DT, Glazer PM, Engelman DM, et al MicroRNA silencing for cancer therapy targeted to the tumour microenvironment. Nature 518: 107-110, 2015.

21. Garofalo $M$ and Croce CM: Role of microRNAs in maintaining cancer stem cells. Adv Drug Deliv Rev 81: 53-61, 2015.

22. Di Leva G, Garofalo M and Croce CM: MicroRNAs in cancer. Annu Rev Pathol 9: 287-314, 2014.

23. Lim EL, Trinh DL, Scott DW, Chu A, Krzywinski M, Zhao Y, Robertson AG, Mungall AJ, Schein J, Boyle M, et al: Comprehensive miRNA sequence analysis reveals survival differences in diffuse large B-cell lymphoma patients. Genome Biol 16: 18, 2015.

24. Yang Y, Xing Y, Liang C, Hu L, Xu F and Chen Y: Crucial microRNAs and genes of human primary breast cancer explored by microRNA-mRNA integrated analysis. Tumour Biol 36 : 5571-5579, 2015.

25. Hata A and Kashima R: Dysregulation of microRNA biogenesis machinery in cancer. Crit Rev Biochem Mol Biol 51: 121-134, 2015.

26. Zhang X, Zhang Y, Yang J, Li S and Chen J: Upregulation of miR-582-5p inhibits cell proliferation, cell cycle progression and invasion by targeting Rab27a in human colorectal carcinoma. Cancer Gene Ther 22: 475-480, 2015.

27. Floyd DH, Zhang Y, Dey BK, Kefas B, Breit H, Marks K, Dutta A, Herold-Mende C, Synowitz M, Glass R, et al: Novel anti-apoptotic microRNAs 582-5p and 363 promote human glioblastoma stem cell survival via direct inhibition of caspase 3 , caspase 9, and Bim. PLoS One 9: e96239, 2014.

28. Jiang J, Zhang Y, Yu C, Li Z, Pan Y and Sun C: MicroRNA-492 expression promotes the progression of hepatic cancer by targeting PTEN. Cancer Cell Int 14: 95, 2014.

29. Peng L, Zhu H, Wang J, Sui H, Zhang H, Jin C, Li L, Xu T and Miao R: miR-492 is functionally involved in oxaliplatin resistance in colon cancer cells LS174T via its regulating the expression of CD147. Mol Cell Biochem 405: 73-79, 2015.
30. Wiggins JF, Ruffino L, Kelnar K, Omotola M, Patrawala L, Brown D and Bader AG: Development of a lung cancer therapeutic based on the tumor suppressor microRNA-34. Cancer Res 70: 5923-5930, 2010.

31. Shimbo K, Miyaki S, Ishitobi H, Kato Y, Kubo T, Shimose S and Ochi M: Exosome-formed synthetic microRNA-143 is transferred to osteosarcoma cells and inhibits their migration. Biochem Biophys Res Commun 445: 381-387, 2014.

32. Tivnan A, Orr WS, Gubala V, Nooney R, Williams DE, McDonagh C, Prenter S, Harvey H, Domingo-Fernández R, Bray IM, et al: Inhibition of neuroblastoma tumor growth by targeted delivery of microRNA-34a using anti-disialoganglioside GD coated nanoparticles. PLoS One 7: e38129, 2012.

33. Wang H, Jiang Y, Peng H, Chen Y, Zhu P and Huang Y: Recent progress in microRNA delivery for cancer therapy by non-viral synthetic vectors. Adv Drug Deliv Rev 81: 142-160, 2015.

34. Kosaka N, Takeshita F, Yoshioka Y, Hagiwara K, Katsuda T, Ono $\mathrm{M}$ and Ochiya T: Exosomal tumor-suppressive microRNAs as novel cancer therapy: 'exocure' is another choice for cancer treatment. Adv Drug Deliv Rev 65: 376-382, 2013.

35. Guo X, Zhang J,Pang J, He S, Li G, Chong Y,Li C, Jiao Z, Zhang S and Shao M: MicroRNA-503 represses epithelial-mesenchymal transition and inhibits metastasis of osteosarcoma by targeting c-myb. Tumour Biol 37: 9181-9187, 2016.

36. Chun-Zhi Z, Lei H, An-Ling Z, Yan-Chao F, Xiao Y, Guang-Xiu W,Zhi-Fan J, Pei-Yu P, Qing-Yu Z and Chun-Sheng K: MicroRNA-221 and microRNA-222 regulate gastric carcinoma cell proliferation and radioresistance by targeting PTEN. BMC Cancer 10: 367, 2010.

37. Ma J, Liu J, Wang Z, Gu X, Fan Y, Zhang W, Xu L, Zhang J and Cai D: NF-kappaB-dependent microRNA-425 upregulation promotes gastric cancer cell growth by targeting PTEN upon IL-1 $\beta$ induction. Mol Cancer 13: 40, 2014.

38. Zhang AX, Lu FQ, Yang YP, Ren XY, Li ZF and Zhang W: MicroRNA-217 overexpression induces drug resistance and invasion of breast cancer cells by targeting PTEN signaling. Cell Biol Int: Jun 24, 2015 (Epub ahead of print).

39. Tang JM, He QY, Guo RX and Chang XJ: Phosphorylated Akt overexpression and loss of PTEN expression in non-small cell lung cancer confers poor prognosis. Lung Cancer 51: 181-191, 2006.

40. Feng B, Zhang K, Wang R and Chen L: Non-small-cell lung cancer and miRNAs: novel biomarkers and promising tools for treatment. Clin Sci (Lond) 128: 619-634, 2015

41. Harris SL and Levine AJ: The p53 pathway: positive and negative feedback loops. Oncogene 24: 2899-2908, 2005. 